\title{
PRELIMINARY EXPERIMENTS WITH SEA URCHIN PLUTEUS AND METAMORPHOSIS IN MARINE POLLUTION BIOASSAY
}

$\operatorname{AUTHOR}(S)$ :

Kobayashi, Naomasa

CITATION:

Kobayashi, Naomasa. PRELIMINARY EXPERIMENTS WITH SEA URCHIN PLUTEUS AND METAMORPHOSIS IN MARINE POLLUTION BIOASSAY. PUBLICATIONS OF THE SETO MARINE BIOLOGICAL LABORATORY 1977, 24(1-3): 9-21

ISSUE DATE:

1977-11-30

URL:

http://hdl.handle.net/2433/175965

RIGHT: 


\title{
PRELIMINARY EXPERIMENTS WITH SEA URGHIN \\ PLUTEUS AND METAMORPHOSIS IN MARINE POLLUTION BIOASSAY
}

\author{
NAOMASA KOBAYASHI \\ Biological Laboratory, Doshisha University, Kyoto
}

With Text-figures 1-12 and Tables 1-5

\section{Introduction}

The author proposed in 1971 the use of sea urchin eggs and embryos as indicatory materials in marine pollution bioassay and actually this was applied to the survey of the sea water pollution in the Inland Sea of Japan (1972). The method was then improved to enhance the sensitivity by using aged eggs (1974). Generally, however, further effects of pollution upon later developmental stages than gastrulation has seemingly been studied only insufficiently in spite of its great importance. Evidently, this is because of the fact that it is rather difficult to rear sea urchin embryos to imagos through their pelagic larval stage and it will take a considerably long time to do this if possible. Therefore, the author tried to examine the effects of some kinds of pollutants upon the formation of pluteus in the sea urchin, Anthocidaris crassispina, and then the effects of some pollutants and polluted water samples upon the whole pelagic life from fertilization to metamorphosis in the sand dollar, Peronella japonica, showing a rapid growth with only a short, three or four days (Mortensen 1921, Onoda 1938, Okazaki and Dan 1954, Okazaki 1975), pelagic life. The present paper is to record the results of those examinations.

\section{Materials and Methods}

1. Examinations in Anthocidaris crassispina

Eggs of Anthocidaris crassispina (A. Agassiz) were inseminated in respective test water samples and rates of fertilization, first cleavage, gastrulation, pluteus formation and some anomalies in development were checked at $26^{\circ} \mathrm{C}$. Eggs were obtained by the current $\mathrm{KCl}$-method, rinsed several times with fresh sea water and experimented with as soon as possible, within 1 hour at the latest. Sperms were obtained from testes within 1 hour after being taken out of the test and used for insemination at the standard density of about 1 dry sperm: 1000 sea water in volume.

1) Contributions from the Seto Marine Biological Laboratory, No. 638.

Publ. Seto Mar. Biol. Lab., XXIV (1/3), 9-21, $1977 . \quad$ (Article 2) 
Firstly, the percent of eggs with the elevated fertilization membrane to the total eggs was read. At the first cleavage occurring mostly about 45 minutes after insemination, the rate and state of the cleavage, in other words proportions of undivided cells, normal two cells and multi-cells caused by polyspermy, were checked. Then, the state of swimming embryos, exclusive of those deposited on the bottom, was checked by reading proportions of permanent blastulae, normal gastrulae and exogastrulae about 12 hours after insemination. Next the state of swimming plutei, except those deposited on the bottom, was checked by seeing proportions of normal and abnormal ones about 24 hours after insemination. In normal plutei at this time, the body contour has clearly changed already from that in previous stages, the digestive tract is completed and spicules are fully developed (Fig. 1). While, in abnormal plutei, the formation of mentioned structures are retarded markedly. In every examination, roughly a hundred eggs or embryos were checked and the examination was repeated 3 times on different batches in respective water samples.

\section{Examinations in Peronella japonica}

The sand dollar, Peronella japonica (Mortensen), is found commonly, though not abundantly, on the shallow sandy bottom in the vicinity of Seto and matures sexually in June to September. The egg, about $300 \mu$ in diameter, is heavily laden with yolk, opaque and pinkish. The number of eggs produced by a female at a time is much fewer than in common sea urchins. In experiments, eggs and sperms were obtained by injection of $\mathrm{KCl}$ through the anus, and the eggs were rinsed several times with fresh sea water and experimented with as soon as possible. The sperm density at insemination was maintained at about 1 dry sperm: 1000 sea water in volume.

Eggs were inseminated in respective test water samples and rates of successful fertilization, first cleavage, gastrulation, pluteus formation and metamorphosis and of some abnormalities in development were checked at $26^{\circ} \mathrm{C}$.

Firstly, the percent of eggs with elevated fertilization membrane to the total eggs was read, this was done 5 minutes after insemination as the elevation of the fertilization membrane was slow in this species. The first cleavage occurred in most cases about 40 minutes after insemination, then the rate and state of the first cleavage, namely proportions of undivided cells, normal two cells and multi-cells caused by polyspermy, were checked. In the two examinations mentioned above, about a hundred eggs were checked respectively. As to the state of swimming embryos, proportions of permanent blastulae, normal gastrulae and exogastrulae were checked in about fifty embryos about 12 hours after insemination. Proportions of abnormal embryos and normal plutei were chekced in about twenty embryos about 24 hours after insemination. And lastly, rates of abnormal larvae and normal imagos were checked in about ten larvae and imagos about 96 hours after insemination. The check was repeated 3 times in every examination on different batches in respective water samples. Before the results of examinations are given, some developmental features in the present sand dollar may be briefly explained. 

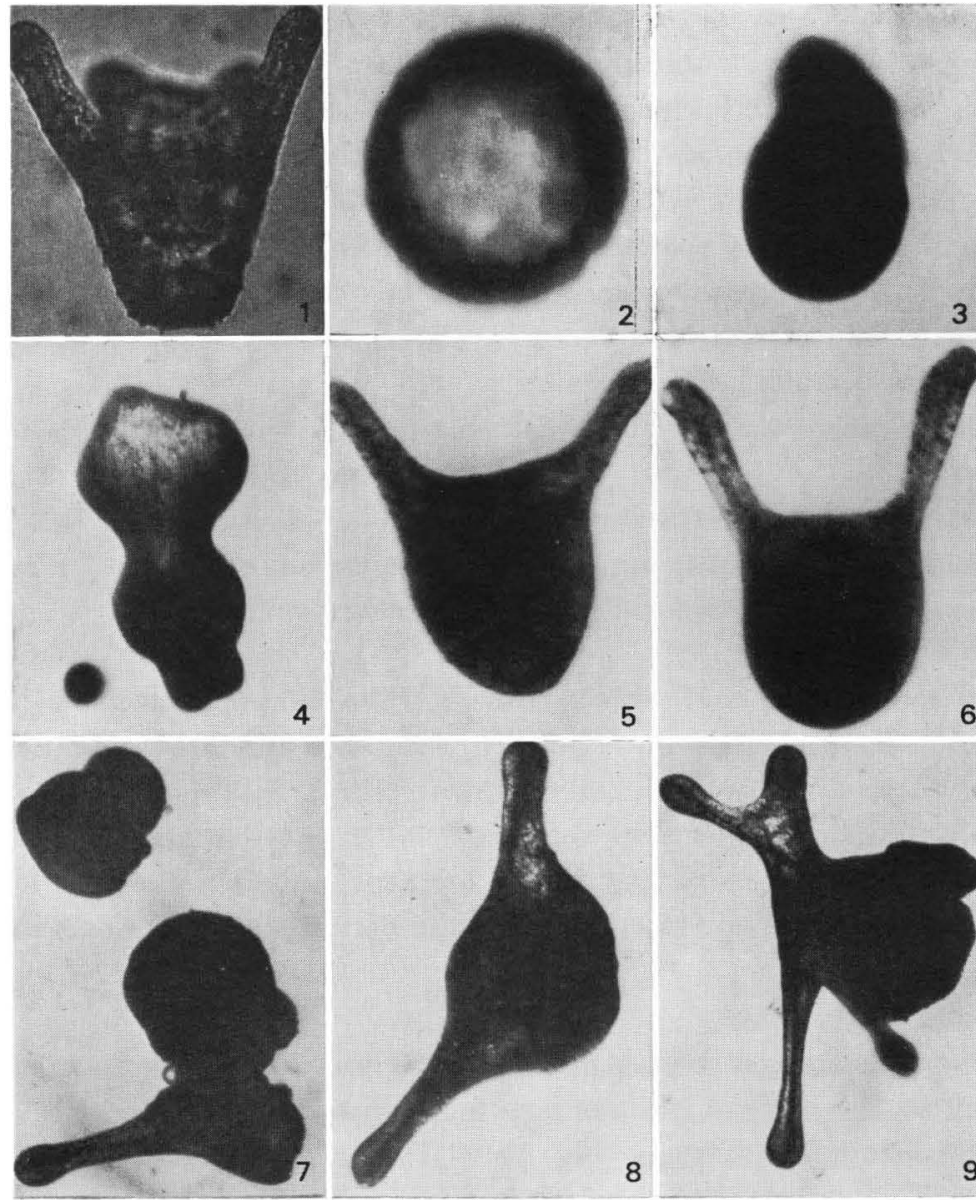

5
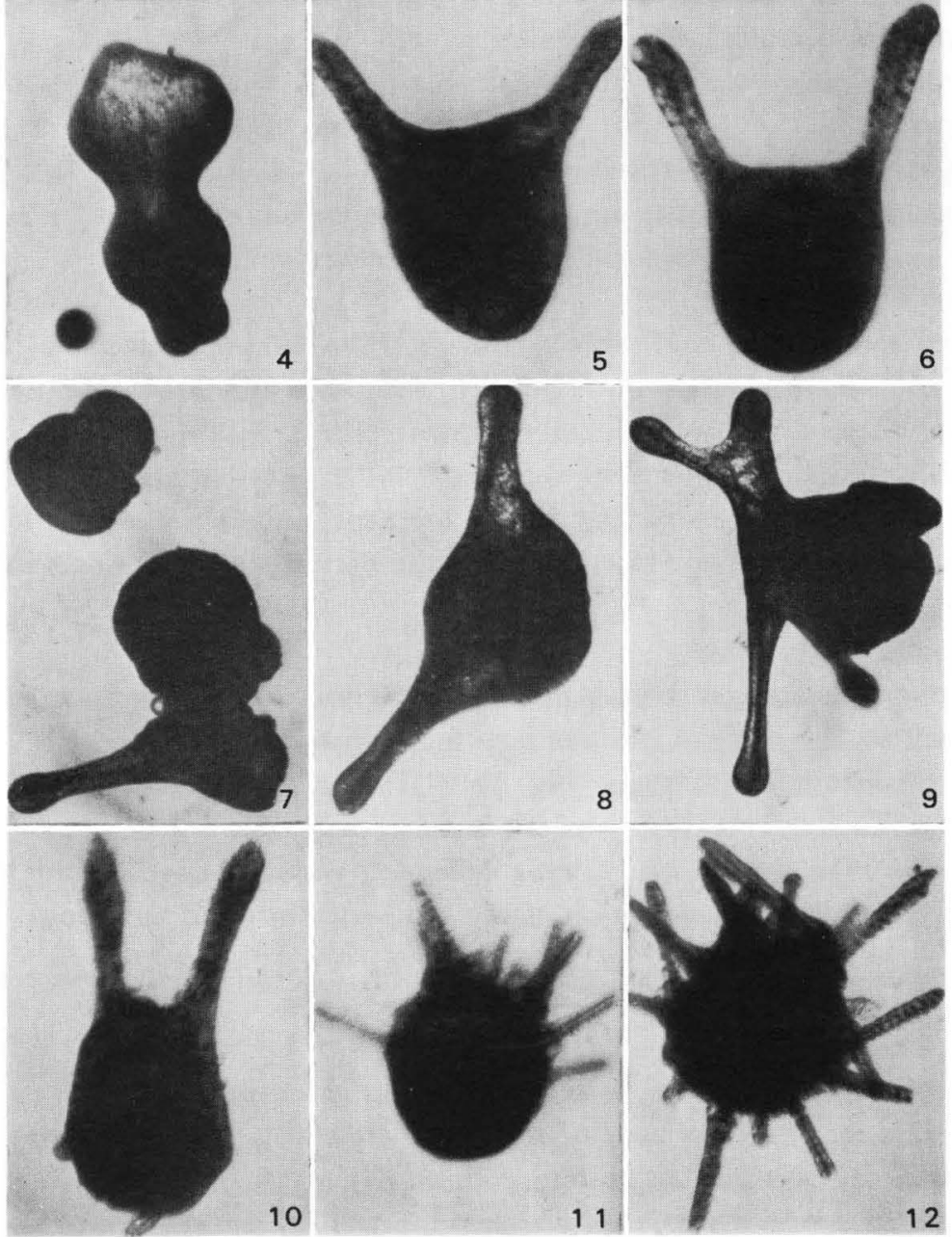

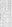
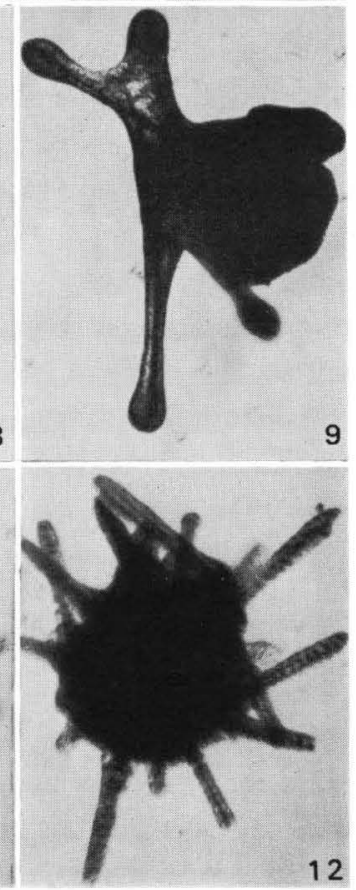

Figs. 1 to 12. Normal and abnormal figures appeared in the developmental stages from blastula to metamorphosis in the experiments with Anthocidaris crassispina and Peronella japonica, $\times 100$. Experiments were made at the water temperature of $26^{\circ} \mathrm{C}$, the normal figures were observed in the running sea water of the Laboratory.

1: Anthocidaris crassispina, normal pluteus, 24 hours after insemination.

2-12: Peronella japonica. 2. Normal blastula, 5 hrs. after insemination. 3. Normal late gastrula, lateral view, $13 \mathrm{hrs}$. after. 4. Exogastrula in the water from Tsunashirazu cove, 18 hrs. after. 5. Normal pluteus, 24 hrs. after. 6. Normal late pluteus, 40 hrs. after. 7-9. Various plutei in the water from Tsunashirazu cove, $80 \mathrm{hrs}$. after. 10. Normal pluteus ready for metamorphosis, 50 hrs. after. 11. Normal imago just after metamorphosis, 70 hrs. after. 12. Normal juvenile, 3 days after metamorphosis. 


\section{(a) Gastrula}

The eggs developing successfully with repeated cleavages will attain the stage from young blastula with folded wall to blastula with expanded wall (Fig. 2) about 5 hours after insemination and then swim out of the fertilization membrane. The elongated embryo looks somewhat like the gastrula of regular sea urchins. Gastrular invagination begins on the posterior wall about 12 hours after insemination. A few hours later, another ingrowth appears at the center of the body and invaginates. The gastrula is formed with such features (Fig. 3, lateral view).

(b) Permanent blastula (Animalization)

The development may be stopped at blastula by some toxic substances, etc. in the sea water.

(c) Exogastrula (Vegetalization)

In gastrulation, the tissue may grow outside the embryo instead of invagination when the sea water contains some toxic substances, etc. (Fig. 4).

(d) Pluteus

Nearly completed plutei with two arms (Fig. 5) appear about 24 hours after insemination, but it takes about 40 hours to reach the fully grown pluteus typical to this species, that is usually furnished with two postal arms (Fig. 6), though the arms may vary in number, from zero to four (Figs. 7-9). Abnormalities in embryos were expressed mainly as the retardation in development in the sea water containing some toxic substances, etc. The pluteus sinks to the bottom about 50 hours after insemination.

(e) Metamorphosis

The metamorphosis of pluteus begins 50-60 hours after insemination, with the protrusion from the body of rudimentary spines and tube feet (Fig. 10), and mostly is completed within 70-80 hours (Fig. 11). Typical six-rayed spines and tube feet are recognizable a few days after metamorphosis (Fig. 12).

Of course, the density of embryos decreased with the development, this might be partly due to the contamination of the test water by dead embryos.

\section{Results}

The concentration of the chemicals tested is given in the tables in ppm of the main effective ion or component of respective chemicals, and their arrangement in tables is made in the order of inhibitory effects.

\section{Effects of some chemicals on developmental stages from fertilization} to pluteus formation in Anthocidaris

The effects of various chemicals upon the developmental stages from fertilization to pluteus formation in Anthocidaris were compared one another. As seen in the results given in Tables 1 and 2, the effects upon the stages from fertilization to gastrulation seemingly conform to those reported already in the previous papers (1971 and 1974), though with some exception. However, by checking pluteus formation additionally and defining the normal development by pluteus formation of $95 \%$ or 
Table 1. Effects of heavy metals upon the developmental stages in Anthocidaris crassispina.

Date: Sept. 5-8. Water temperature: $26^{\circ} \mathrm{C}$.

\begin{tabular}{|c|c|c|c|c|c|c|c|c|c|c|c|}
\hline \multirow{2}{*}{ Chemicals } & \multirow{2}{*}{$\begin{array}{r}\text { Concent- } \\
\text { rations } \\
\text { ppm }\end{array}$} & \multirow{2}{*}{\begin{tabular}{l|}
\multicolumn{1}{c|}{3 -min. } \\
after insemi. \\
fertilization \\
membrane \\
formation \%
\end{tabular}} & \multicolumn{3}{|c|}{$45 \mathrm{~min}$. after insemination } & \multicolumn{3}{|c|}{$12 \mathrm{hrs}$. after insemination } & \multicolumn{2}{|c|}{$\begin{array}{l}24 \mathrm{hrs} \text {. after } \\
\text { insemination }\end{array}$} & \multirow{2}{*}{ Ultimate state } \\
\hline & & & $\begin{array}{l}\text { 1-cell } \\
\text { state } \\
\end{array}$ & $\begin{array}{l}2 \text {-cell } \\
\text { state } \\
\end{array}$ & $\begin{array}{l}\text { multi-cell, } \\
\text { polyspermy } \\
\%\end{array}$ & $\begin{array}{l}\text { permanent } \\
\text { blastula } \\
\end{array}$ & $\begin{array}{r}\text { normal } \\
\text { gastrula } \\
\%\end{array}$ & $\begin{array}{l}\text { exo- } \\
\text { gastrula } \\
\%\end{array}$ & \begin{tabular}{|l|}
$\begin{array}{l}\text { abnormal } \\
\text { pluteus } \\
\%\end{array}$ \\
\end{tabular} & $\begin{array}{r}\text { normal } \\
\text { pluteus } \\
\%\end{array}$ & \\
\hline Control water & & 100 & 5 & 95 & 0 & 0 & 100 & 0 & 0 & 100 & normal \\
\hline $\mathrm{HgCl}_{2}$ & $\begin{array}{l}0.5 \\
0.25 \\
0.12 \\
0.06 \\
0.03 \\
0.015\end{array}$ & $\begin{array}{r}15 \\
60 \\
85 \\
90 \\
95 \\
100\end{array}$ & $\begin{array}{r}100 \\
55 \\
30 \\
15 \\
10 \\
5\end{array}$ & $\begin{array}{r}0 \\
45 \\
70 \\
85 \\
90 \\
95\end{array}$ & $\begin{array}{l}0 \\
0 \\
0 \\
0 \\
0 \\
0\end{array}$ & $\begin{array}{r}80 \\
55 \\
30 \\
0\end{array}$ & $\begin{array}{r}20 \\
45 \\
70 \\
100\end{array}$ & $\begin{array}{l}0 \\
0 \\
0 \\
0\end{array}$ & $\begin{array}{r}40 \\
5\end{array}$ & $\begin{array}{l}60 \\
95\end{array}$ & $\begin{array}{l}\text { cytolysis } \\
4-8 \text { cell } \\
\text { permanent blastula } \\
\text { retardation } \\
\text { retardation } \\
\text { normal }\end{array}$ \\
\hline $\mathrm{CuSO}_{4} \cdot 5 \mathrm{H}_{2} \mathrm{O}$ & $\begin{array}{l}1 \\
0.5 \\
0.25 \\
0.12 \\
0.06 \\
0.03\end{array}$ & $\begin{array}{r}0 \\
55 \\
85 \\
95 \\
100 \\
100\end{array}$ & $\begin{array}{r}100 \\
50 \\
15 \\
15 \\
0 \\
0\end{array}$ & $\begin{array}{r}0 \\
40 \\
70 \\
40 \\
100 \\
100\end{array}$ & $\begin{array}{r}0 \\
10 \\
15 \\
45 \\
0 \\
0\end{array}$ & $\begin{array}{r}100 \\
30 \\
0 \\
0 \\
0\end{array}$ & $\begin{array}{r}0 \\
70 \\
100 \\
100 \\
100\end{array}$ & $\begin{array}{l}0 \\
0 \\
0 \\
0 \\
0\end{array}$ & $\begin{array}{r}100 \\
100 \\
10 \\
0\end{array}$ & $\begin{array}{r}0 \\
0 \\
90 \\
100\end{array}$ & $\begin{array}{l}\text { unfertilized } \\
\text { permanent blastula } \\
\text { retardation } \\
\text { retardation } \\
\text { retardation } \\
\text { normal }\end{array}$ \\
\hline $\mathrm{ZnCl}_{2}$ & $\begin{array}{l}1 \\
0.5 \\
0.25 \\
0.12 \\
0.06 \\
0.03\end{array}$ & $\begin{array}{r}0 \\
50 \\
100 \\
100 \\
100 \\
100\end{array}$ & $\begin{array}{r}100 \\
50 \\
0 \\
0 \\
0 \\
0\end{array}$ & $\begin{array}{r}0 \\
45 \\
85 \\
90 \\
100 \\
100\end{array}$ & $\begin{array}{r}5 \\
15 \\
10 \\
0 \\
0\end{array}$ & $\begin{array}{r}50 \\
30 \\
30 \\
10 \\
0\end{array}$ & $\begin{array}{r}5 \\
40 \\
70 \\
90 \\
100\end{array}$ & $\begin{array}{r}45 \\
30 \\
0 \\
0 \\
0\end{array}$ & $\begin{array}{r}80 \\
40 \\
10 \\
0\end{array}$ & $\begin{array}{r}20 \\
60 \\
90 \\
100\end{array}$ & $\begin{array}{l}\text { unfertilized } \\
\text { exogastrulation } \\
\text { retardation } \\
\text { retardation } \\
\text { retardation } \\
\text { normal }\end{array}$ \\
\hline $\mathrm{CdCl}_{2} \cdot 2 \frac{1}{2} \mathrm{H}_{2} \mathrm{O}$ & $\begin{array}{l}25 \\
12 \\
6 \\
3 \\
1.5\end{array}$ & $\begin{array}{r}0 \\
40 \\
90 \\
100 \\
100\end{array}$ & $\begin{array}{r}100 \\
65 \\
15 \\
0 \\
0\end{array}$ & $\begin{array}{r}0 \\
35 \\
85 \\
100 \\
100\end{array}$ & $\begin{array}{l}0 \\
0 \\
0 \\
0 \\
0\end{array}$ & $\begin{array}{r}100 \\
20 \\
0\end{array}$ & $\begin{array}{r}0 \\
80 \\
100\end{array}$ & $\begin{array}{l}0 \\
0 \\
0\end{array}$ & $\begin{array}{r}100 \\
0\end{array}$ & $\begin{array}{r}0 \\
100\end{array}$ & $\begin{array}{l}\text { unfertilized } \\
4-8 \text { cells } \\
\text { permanent blastula } \\
\text { retardation } \\
\text { normal }\end{array}$ \\
\hline $\mathrm{K}_{2} \mathrm{Cr}_{2} \mathrm{O}_{7}$ & $\begin{array}{r}50 \\
25 \\
12 \\
6 \\
3\end{array}$ & $\begin{array}{r}15 \\
50 \\
90 \\
95 \\
100\end{array}$ & $\begin{array}{r}90 \\
55 \\
15 \\
5 \\
0\end{array}$ & $\begin{array}{r}10 \\
45 \\
85 \\
95 \\
100\end{array}$ & $\begin{array}{l}0 \\
0 \\
0 \\
0 \\
0\end{array}$ & $\begin{array}{r}70 \\
0 \\
5 \\
0\end{array}$ & $\begin{array}{r}30 \\
100 \\
95 \\
100\end{array}$ & $\begin{array}{l}0 \\
0 \\
0 \\
0\end{array}$ & $\begin{array}{r}70 \\
15 \\
10 \\
0\end{array}$ & $\begin{array}{r}30 \\
85 \\
90 \\
100\end{array}$ & $\begin{array}{l}1-4 \text { cells } \\
\text { retardation } \\
\text { retardation } \\
\text { retardation } \\
\text { normal }\end{array}$ \\
\hline
\end{tabular}


Table 2. Effects of various chemicals upon the developmental stages in Anthocidaris crassispina.

Date: Sept. 5-8. Water temperature: $26^{\circ} \mathrm{C}$.

\begin{tabular}{|c|c|c|c|c|c|c|c|c|c|c|c|}
\hline \multirow{2}{*}{ Chemicals } & \multirow{2}{*}{$\begin{array}{l}\text { Concent- } \\
\text { rations } \\
\text { ppm }\end{array}$} & \multirow{2}{*}{\begin{tabular}{l}
\multicolumn{1}{c|}{3 -min. } \\
after insemi.
\end{tabular}} & \multicolumn{3}{|c|}{$45 \mathrm{~min}$. after insemination } & \multicolumn{3}{|c|}{$12 \mathrm{hrs}$. after insemination } & \multicolumn{2}{|c|}{$\begin{array}{l}24 \mathrm{hrs.} \mathrm{after} \\
\text { insemination }\end{array}$} & \multirow{2}{*}{ Ultimate state } \\
\hline & & & 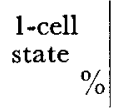 & $\begin{array}{l}\text { 2-cell } \\
\text { state } \\
\end{array}$ & $\begin{array}{l}\text { multi-cell, } \\
\text { polyspermy } \\
\%\end{array}$ & \begin{aligned} & \multicolumn{2}{c|}{ permanent } \\
& blastula \\
&$\%\end{aligned}$ & $\begin{array}{r}\text { normal } \\
\text { gastrula } \\
\%\end{array}$ & \begin{tabular}{|l|} 
exo- \\
gastrula \\
$\%$
\end{tabular} & $\mid \begin{array}{l}\text { abnormal } \\
\text { pluteus } \\
\%\end{array}$ & $\begin{array}{r}\text { normal } \\
\text { pluteus } \\
\%\end{array}$ & \\
\hline Control water & & 100 & 0 & 100 & 0 & 0 & 100 & 0 & 0 & 100 & normal \\
\hline \multirow{5}{*}{ ABS } & 12 & 0 & 100 & 0 & 0 & & & & & & unfertilized \\
\hline & 6 & 10 & 95 & 5 & 0 & & & & & & cytolysis \\
\hline & 3 & 35 & 80 & 20 & 0 & 40 & 60 & 0 & 100 & 0 & retardation \\
\hline & 1.5 & 95 & 10 & 90 & 0 & 10 & 90 & 0 & 20 & 80 & retardation \\
\hline & 0.8 & 95 & 10 & 90 & 0 & 5 & 95 & 0 & 0 & 100 & normal \\
\hline \multirow{6}{*}{$\mathrm{NH}_{4} \mathrm{Cl}$} & 50 & 0 & 100 & 0 & 0 & & & & & & unfertilized \\
\hline & 25 & 10 & 95 & 5 & 0 & & & & & & cytolysis \\
\hline & 12 & 60 & 50 & 50 & 0 & 100 & 0 & 0 & & & permanent blastula \\
\hline & 6 & 80 & 25 & 75 & 0 & 30 & 70 & 0 & 100 & 0 & retardation \\
\hline & 3 & 85 & 25 & 75 & 0 & 10 & 90 & 0 & 45 & 55 & retardation \\
\hline & 1.5 & 90 & 10 & 90 & 0 & 0 & 100 & 0 & 0 & 100 & normal \\
\hline \multirow{6}{*}{$\mathrm{As}_{2} \mathrm{O}_{5}$} & 50 & 0 & 100 & 0 & 0 & & & & & & unfertilized \\
\hline & 25 & 60 & 50 & 50 & 0 & 100 & 0 & 0 & & & permanent blastula \\
\hline & 12 & 80 & 30 & 70 & 0 & 70 & 30 & 0 & 100 & 0 & retardation \\
\hline & 6 & 90 & 25 & 75 & 0 & 25 & 75 & 0 & 95 & 5 & retardation \\
\hline & 3 & 100 & 10 & 90 & 0 & 10 . & 90 & 0 & 45 & 55 & retardation \\
\hline & 1.5 & 100 & 0 & 100 & 0 & 0 & 100 & 0 & 5 & 95 & normal \\
\hline
\end{tabular}


more of normal gastrulae, the malefic effects of these chemicals are perceived more strictly (by about one grade of concentration in respective chemicals).

(1) Effects of heavy metals

In solutions of most compounds except cadmium chloride, their effects were seen more pronouncedly in pluteus formation. Generally speaking, heavy metals seem to affect the pluteus formation more strongly than the stages prior to it.

(2) Effects of chemicals other than heavy metals

As in the case of heavy metals, the effects seemed more pronounced in pluteus formation in solutions of all compounds tested, at least the effects upon the pluteus formation were seemingly larger than upon the gastrulation.

Effects of some chemicals on developmental stages from fertilization to metamorphosis in Peronella

The effects of various chemicals upon the developmental stages from fertilization to metamorphosis in Peronella are given in Tables 3 and 4 . Generally seeing, the developmental stages in Peronella seem more sensitive to tested chemicals than in Anthocidaris, especially the metamorphosis is seemingly affected much more sensitively by them than the preceding stages. In the case of this sand dollar, the normal development in the present examination was defined by the pluteus formation of $85 \%$ or more of gastrulae and the successful metamorphosis of $70 \%$ or more of plutei.

(1) Effects of heavy metals

In solutions of all heavy metals tested, the effects on plutei seemed more pronounced than in Anthocidaris; this was especially remarkable in the case of potassium dichromate. Further, the metamorphosis was affected by every tested heavy metal much more (by more than two grades of concentration in respective chemicals) sensitively than plutei.

(2) Effects of other chemicals

The effects on plutei seemed more pronounced than in Anthocidaris in solutions of all chemicals, especially of arsenic acid pentoxide. The metamorphosis was affected by them more sensitively than plutei, especially in the case of arsenic pentoxide.

Effects of the polluted sea water from around Hatakejima Island, tested by developmental features of Peronella

The effects of the polluted sea water were learned by comparing the figures of developing embryos or larvae of Peronella in the water with those in the control water, the running sea water of the laboratory. The samples of polluted sea water were collected at the two stations respectively around and near Hatakejima Island in Tanabe Bay, that were shown already in previous papers (Kobayashi 1971 and 1974). It was found that the effects became larger with the development and more pronounced with the progress of pollution (Table 5). Really the development was affected most markedly in the water samples from the cove of Tsunashirazu where the water was polluted most heavily by sweage and waste products of fish rearing. Throughout the whole developmental stages from fertilization to pluteus formation, Peronella was affected by the polluted water more sensitively than Anthocidaris. 
Table 3. Effects of heavy metals upon the developmental stages in Peronella japonica.

Date: Sept. 2-8. Water temperature: $26^{\circ} \mathrm{C}$.

\begin{tabular}{|c|c|c|c|c|c|c|c|c|c|c|c|c|c|}
\hline \multirow[b]{2}{*}{ Chemicals } & \multirow{2}{*}{\begin{tabular}{|} 
Concent- \\
rations \\
\\
$\mathrm{ppm}$
\end{tabular}} & \multirow{2}{*}{$\begin{array}{l}\quad \text { 5-min. } \\
\text { after insemi. } \\
\text { fertilization } \\
\text { membrane } \\
\text { formation } \%\end{array}$} & \multicolumn{3}{|c|}{$40 \mathrm{~min}$. after insemi. } & \multicolumn{3}{|c|}{$12 \mathrm{hrs}$. after insemination } & \multicolumn{2}{|c|}{$\begin{array}{l}24 \text { hrs. after } \\
\text { insemination }\end{array}$} & \multicolumn{2}{|c|}{$\begin{array}{l}96 \mathrm{hrs} \text {. after } \\
\text { insemination }\end{array}$} & \multirow[b]{2}{*}{ Ultimate state } \\
\hline & & & \begin{tabular}{|}
$1-$ cell \\
state \\
$\%$
\end{tabular} & $\begin{array}{c}2 \text {-cell } \\
\text { state } \\
\%\end{array}$ & $\mid \begin{array}{r}\text { multi-cell, } \\
\text { polyspermy } \\
\%\end{array}$ & $\begin{array}{r}\text { permanent } \\
\text { blastula } \\
\%\end{array}$ & \begin{tabular}{|r|} 
normal \\
gastrula \\
$\%$
\end{tabular} & \begin{tabular}{|r|}
$\begin{array}{l}\text { exo- } \\
\text { gastrula }\end{array}$ \\
$\%$
\end{tabular} & $\begin{array}{c}\begin{array}{c}\text { abnormal } \\
\text { embryo } \\
\end{array} \\
\end{array}$ & \begin{tabular}{|r|} 
normal \\
pluteus \\
$\%$
\end{tabular} & \begin{tabular}{|l|} 
abnormal \\
larva \\
\end{tabular} & $\begin{array}{c}\text { normal } \\
\text { imago } \\
\%\end{array}$ & \\
\hline $\begin{array}{c}\text { Control } \\
\text { water }\end{array}$ & & 95 & 5 & 95 & 0 & 5 & 95 & 0 & 15 & 85 & 20 & 80 & normal \\
\hline $\mathrm{HgCl}_{2}$ & \begin{tabular}{l|}
0.5 \\
0.25 \\
0.12 \\
0.06 \\
0.03 \\
0.015 \\
0.008
\end{tabular} & $\begin{array}{r}0 \\
30 \\
50 \\
75 \\
90 \\
95 \\
95\end{array}$ & $\begin{array}{r}75 \\
55 \\
25 \\
10 \\
5 \\
5\end{array}$ & $\begin{array}{l}25 \\
45 \\
75 \\
90 \\
95 \\
95\end{array}$ & $\begin{array}{l}0 \\
0 \\
0 \\
0 \\
0 \\
0\end{array}$ & $\begin{array}{r}75 \\
30 \\
10 \\
5\end{array}$ & $\begin{array}{l}25 \\
70 \\
90 \\
95\end{array}$ & $\begin{array}{l}0 \\
0 \\
0 \\
0\end{array}$ & $\begin{array}{r}65 \\
30 \\
5\end{array}$ & $\begin{array}{l}35 \\
70 \\
95\end{array}$ & $\begin{array}{l}90 \\
50\end{array}$ & $\begin{array}{l}10 \\
50\end{array}$ & $\begin{array}{l}\text { unfertilized } \\
2-4 \text { cells } \\
4-8 \text { cells } \\
\text { permanent blastula } \\
\text { retardation } \\
\text { retardation } \\
\text { almost normal }\end{array}$ \\
\hline $\begin{array}{r}\mathrm{CuSO}_{4} \\
5 \mathrm{H}_{2} \mathrm{O}\end{array}$ & $\begin{array}{l}0.5 \\
0.25 \\
0.12 \\
0.06 \\
0.03 \\
0.015\end{array}$ & $\begin{array}{r}0 \\
10 \\
30 \\
85 \\
95 \\
95\end{array}$ & $\begin{array}{r}95 \\
75 \\
15 \\
5 \\
5\end{array}$ & $\begin{array}{r}5 \\
25 \\
85 \\
95 \\
95\end{array}$ & $\begin{array}{l}0 \\
0 \\
0 \\
0 \\
0\end{array}$ & $\begin{array}{l}90 \\
40 \\
20 \\
10\end{array}$ & $\begin{array}{l}10 \\
60 \\
80 \\
90\end{array}$ & $\begin{array}{l}0 \\
0 \\
0 \\
0\end{array}$ & $\begin{array}{l}55 \\
30 \\
15\end{array}$ & $\begin{array}{l}45 \\
70 \\
85\end{array}$ & $\begin{array}{l}80 \\
40\end{array}$ & $\begin{array}{l}20 \\
60\end{array}$ & $\begin{array}{l}\text { unfertilized } \\
16-32 \text { cells } \\
\text { permanent blastula } \\
\text { retardation } \\
\text { retardation } \\
\text { almost normal }\end{array}$ \\
\hline $\mathrm{ZnCl}_{2}$ & \begin{tabular}{l|}
0.5 \\
0.25 \\
0.12 \\
0.06 \\
0.03 \\
0.015
\end{tabular} & $\begin{array}{l}15 \\
30 \\
60 \\
70 \\
90 \\
95\end{array}$ & $\begin{array}{r}100 \\
85 \\
50 \\
40 \\
15 \\
10\end{array}$ & $\begin{array}{r}0 \\
15 \\
50 \\
60 \\
85 \\
90\end{array}$ & $\begin{array}{l}0 \\
0 \\
0 \\
0 \\
0 \\
0\end{array}$ & $\begin{array}{l}85 \\
40 \\
15 \\
10\end{array}$ & $\begin{array}{l}15 \\
60 \\
85 \\
90\end{array}$ & $\begin{array}{l}0 \\
0 \\
0 \\
0\end{array}$ & $\begin{array}{r}100 \\
40 \\
20\end{array}$ & $\begin{array}{r}0 \\
60 \\
80\end{array}$ & $\begin{array}{l}85 \\
30\end{array}$ & $\begin{array}{l}15 \\
70\end{array}$ & $\begin{array}{l}1 \text { cell } \\
2-4 \text { cells } \\
\text { permanent blastula } \\
\text { retardation } \\
\text { retardation } \\
\text { almost normal }\end{array}$ \\
\hline$\underset{2 \frac{1}{2} \mathrm{H}_{2} \mathrm{O}}{\mathrm{CdCl}_{2} \cdot}$ & $\begin{array}{l}20 \\
10 \\
5 \\
2.5 \\
1.2 \\
0.6\end{array}$ & $\begin{array}{l}20 \\
70 \\
90 \\
90 \\
90 \\
95\end{array}$ & $\begin{array}{r}80 \\
35 \\
15 \\
10 \\
10 \\
5\end{array}$ & $\begin{array}{l}20 \\
65 \\
85 \\
90 \\
90 \\
95\end{array}$ & $\begin{array}{l}0 \\
0 \\
0 \\
0 \\
0 \\
0\end{array}$ & $\begin{array}{r}100 \\
100 \\
45 \\
20 \\
10\end{array}$ & $\begin{array}{r}0 \\
0 \\
65 \\
80 \\
90\end{array}$ & $\begin{array}{l}0 \\
0 \\
0 \\
0 \\
0\end{array}$ & $\begin{array}{l}65 \\
25 \\
10\end{array}$ & $\begin{array}{l}35 \\
75 \\
90\end{array}$ & $\begin{array}{l}90 \\
35\end{array}$ & $\begin{array}{l}10 \\
65\end{array}$ & $\begin{array}{l}1-2 \text { cells } \\
\text { permanent blastula } \\
\text { permanent blastula } \\
\text { retardation } \\
\text { retardation } \\
\text { almost normal }\end{array}$ \\
\hline $\mathrm{K}_{2} \mathrm{Cr}_{2} \mathrm{O}_{7}$ & $\begin{array}{l}20 \\
10 \\
5 \\
2.5 \\
1.2 \\
0.6\end{array}$ & $\begin{array}{r}0 \\
30 \\
45 \\
80 \\
90 \\
95\end{array}$ & $\begin{array}{r}70 \\
60 \\
20 \\
15 \\
5\end{array}$ & $\begin{array}{l}30 \\
40 \\
80 \\
85 \\
95\end{array}$ & $\begin{array}{l}0 \\
0 \\
0 \\
0 \\
0\end{array}$ & $\begin{array}{r}40 \\
20 \\
10 \\
5\end{array}$ & $\begin{array}{l}60 \\
80 \\
90 \\
95\end{array}$ & $\begin{array}{l}0 \\
0 \\
0 \\
0\end{array}$ & $\begin{array}{r}100 \\
100 \\
35 \\
15\end{array}$ & $\begin{array}{r}0 \\
0 \\
65 \\
85\end{array}$ & $\begin{array}{l}45 \\
25\end{array}$ & $\begin{array}{l}55 \\
75\end{array}$ & $\begin{array}{l}\text { unfertilized } \\
1-4 \text { cells } \\
\text { retardation } \\
\text { retardation } \\
\text { almost normal } \\
\text { normal }\end{array}$ \\
\hline
\end{tabular}


Table 4. Effects of various chemicals upon the developmental stages in Peronella japonica.

Date: Sept. 2-8. Water temperature: $26^{\circ} \mathrm{C}$.

\begin{tabular}{|c|c|c|c|c|c|c|c|c|c|c|c|c|c|}
\hline \multirow[b]{2}{*}{ Chemicals } & \multirow{2}{*}{$\begin{array}{l}\text { Concent- } \\
\text { rations }\end{array}$} & \multirow{2}{*}{ 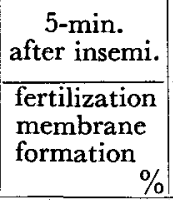 } & \multicolumn{3}{|c|}{$40 \mathrm{~min}$. after insemi. } & \multicolumn{3}{|c|}{$12 \mathrm{hrs}$. after insemination } & \multicolumn{2}{|c|}{$\begin{array}{l}24 \mathrm{hrs.} \mathrm{after} \\
\text { insemination }\end{array}$} & \multicolumn{2}{|c|}{$\begin{array}{l}96 \mathrm{hrs} \text {. after } \\
\text { insemination }\end{array}$} & \multirow[b]{2}{*}{ Ultimate state } \\
\hline & & & $\begin{array}{r}\text { 1-cell } \\
\text { state } \\
\%\end{array}$ & $\begin{array}{c}\text { 2-cell } \\
\text { state } \\
\%\end{array}$ & $\begin{array}{r}\text { multi-cell, } \\
\text { polyspermy } \\
\%\end{array}$ & $\begin{array}{r}\text { permanent } \\
\text { blastula } \\
\%\end{array}$ & \begin{tabular}{|} 
normal \\
gastrula \\
$\%$
\end{tabular} & $\begin{array}{r}\text { exo- } \\
\text { gastrula } \\
\%\end{array}$ & $\begin{array}{r}\text { abnormal } \\
\text { embryo } \\
\%\end{array}$ & $\begin{array}{r}\text { normal } \\
\text { pluteus } \\
\%\end{array}$ & $\begin{array}{l}\begin{array}{l}\text { abnormal } \\
\text { larva }\end{array} \\
\%\end{array}$ & 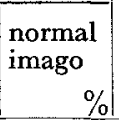 & \\
\hline $\begin{array}{c}\begin{array}{c}\text { Control } \\
\text { water }\end{array} \\
\end{array}$ & & 95 & 10 & 90 & 0 & 5 & 95 & 0 & 10 & 90 & 20 & 80 & normal \\
\hline \multirow{9}{*}{$\mathrm{As}_{2} \mathrm{O}_{5}$} & 50 & 0 & & & & & & & & & & & unfertilized \\
\hline & 25 & 50 & 60 & 40 & 0 & 100 & 0 & 0 & & & & & cytolysis \\
\hline & 12 & 75 & 40 & 60 & 0 & 85 & 15 & 0 & & & & & permanent blastula \\
\hline & 6 & 90 & 15 & 85 & 0 & 60 & 40 & 0 & 100 & 0 & & & retardation \\
\hline & 3 & 90 & 15 & 85 & 0 & 30 & 70 & 0 & 100 & 0 & & & retardation \\
\hline & 1.5 & 95 & 10 & 90 & 0 & 20 & 80 & 0 & 65 & 35 & 100 & 0 & retardation \\
\hline & 0.8 & 95 & 10 & 90 & 0 & 10 & 90 & 0 & 20 & 80 & 100 & 0 & retardation \\
\hline & 0.4 & 95 & 5 & 95 & 0 & 5 & 95 & 0 & 20 & 80 & 70 & 30 & retardation \\
\hline & 0.2 & 95 & 5 & 95 & 0 & 5 & 95 & 0 & 10 & 90 & 20 & 80 & normal \\
\hline \multirow{6}{*}{ ABS } & 10 & 10 & 95 & 5 & 0 & & & & & & & & cytolysis \\
\hline & 5 & 25 & 85 & 15 & 0 & 100 & 0 & 0 & & & & & permanent blastula \\
\hline & 2.5 & 80 & 20 & 80 & 0 & 30 & 70 & 0 & 100 & 0 & & & retardation \\
\hline & 1.2 & 95 & 10 & 90 & 0 & 15 & 85 & 0 & 100 & 0 & 100 & 0 & retardation \\
\hline & 0.6 & 95 & 10 & 90 & 0 & 10 & 90 & 0 & 20 & 80 & 80 & 20 & retardation \\
\hline & 0.3 & 95 & 5 & 95 & 0 & 5 & 95 & 0 & 10 & 90 & 30 & 70 & normal \\
\hline \multirow{6}{*}{$\mathrm{NH}_{4} \mathrm{Gl}$} & 20 & 0 & & & & & & & & & & & unfertilized \\
\hline & 10 & 40 & 70 & 30 & 0 & 100 & 0 & 0 & & & & & permanent blastula \\
\hline & 5 & 85 & 15 & 85 & 0 & 40 & 55 & 5 & 100 & 0 & & & retardation \\
\hline & 2.5 & 90 & 10 & 90 & 0 & 20 & 75 & 5 & 65 & 35 & 100 & 0 & retardation \\
\hline & 1.2 & 95 & 10 & 90 & 0 & 10 & 90 & 0 & 15 & 85 & 90 & 10 & retardation \\
\hline & 0.6 & 95 & 5 & 95 & 0 & 5 & 95 & 0 & 10 & 90 & 30 & 70 & normal \\
\hline
\end{tabular}


Table 5. Effects of polluted sea water from around Hatakejima Island on the developmental stages in Peronella japonica. Date: Sept. 3-8. Water temperature: $26^{\circ} \mathrm{C}$. *: Eggs from the same batch were used. **: Eggs in three examinations came respectively from the same batches.

\begin{tabular}{|c|c|c|c|c|c|c|c|c|c|c|c|c|c|}
\hline \multirow[b]{2}{*}{ Location } & \multirow[b]{2}{*}{ (depth) } & \multirow{2}{*}{\begin{tabular}{|l|}
\multicolumn{1}{c|}{5 -min. } \\
after insemi. \\
fertilization \\
membrane \\
formation
\end{tabular}} & \multicolumn{3}{|c|}{45 min. after insemi. } & \multicolumn{3}{|c|}{12 hrs. after insemi. } & \multicolumn{2}{|c|}{24 hrs. after insemi. } & \multicolumn{2}{|c|}{$96 \mathrm{hrs}$. after insemi. } & \multirow{2}{*}{$\begin{array}{l}\text { Ultimate } \\
\text { state }\end{array}$} \\
\hline & & & $\begin{array}{c}1-\text { cell } \\
\text { state } \\
\%\end{array}$ & $\begin{array}{l}2 \text {-cell } \\
\text { state } \\
\%\end{array}$ & $\begin{array}{l}\text { multi-cell, } \\
\text { polyspermy } \\
\%\end{array}$ & $\begin{array}{r}\text { permanent } \\
\text { blastula } \\
\%\end{array}$ & $\begin{array}{r}\text { normal } \\
\text { gastrula } \\
\%\end{array}$ & $\begin{array}{r}\text { exo- } \\
\text { gastrula } \\
\%\end{array}$ & $\begin{array}{l}\text { abnormal } \\
\text { embryo } \\
\%\end{array}$ & $\begin{array}{r}\text { normal } \\
\text { pluteus } \\
\%\end{array}$ & $\begin{array}{r}\text { abnormal } \\
\text { larva } \\
\%\end{array}$ & $\begin{array}{r}\text { normal } \\
\text { imago } \\
\%\end{array}$ & \\
\hline $\begin{array}{l}\text { Running sea } \\
\text { water of } \\
\text { Laboratory }\end{array}$ & & $* *\left(\begin{array}{l}100 \\
100 \\
100 \\
100\end{array}\right.$ & $\begin{array}{r}5 \\
10 \\
5 \\
10\end{array}$ & $\begin{array}{l}95 \\
90 \\
95 \\
90\end{array}$ & $\begin{array}{l}0 \\
0 \\
0 \\
0\end{array}$ & $\begin{array}{r}5 \\
10 \\
0 \\
0\end{array}$ & $\begin{array}{r}95 \\
90 \\
100 \\
100\end{array}$ & $\begin{array}{l}0 \\
0 \\
0 \\
0\end{array}$ & $\begin{array}{r}5 \\
10 \\
5 \\
10\end{array}$ & $\begin{array}{l}95 \\
90 \\
95 \\
90\end{array}$ & $\begin{array}{l}30 \\
25 \\
25 \\
20\end{array}$ & $\begin{array}{l}70 \\
75 \\
75 \\
80\end{array}$ & $\begin{array}{l}\text { normal } \\
\text { normal } \\
\text { normal } \\
\text { normal }\end{array}$ \\
\hline $\begin{array}{l}\text { Water from } \\
\text { land side of } \\
\text { Hatakejima }\end{array}$ & $\begin{array}{l}\text { Bottom } \\
(25 \mathrm{~m})\end{array}$ & $* 100$ & 10 & 90 & 0 & 30 & 70 & 0 & 85 & 15 & 100 & 0 & retardation \\
\hline $\begin{array}{l}\text { Water from } \\
\text { Tsunashirazu } \\
\text { cove }\end{array}$ & Surface & $* 80$ & 25 & 75 & 0 & 65 & 35 & 0 & 100 & 0 & 100 & 0 & retardation \\
\hline $\begin{array}{l}\text { Water from } \\
\text { Tsunashirazu } \\
\text { cove }\end{array}$ & $\begin{array}{c}\text { Bottom } \\
(5 \mathrm{~m})\end{array}$ & $* *\left(\begin{array}{r}* 90 \\
85 \\
100 \\
100\end{array}\right.$ & $\begin{array}{l}15 \\
25 \\
10 \\
10\end{array}$ & $\begin{array}{l}85 \\
75 \\
90 \\
90\end{array}$ & $\begin{array}{l}0 \\
0 \\
0 \\
0\end{array}$ & $\begin{array}{r}45 \\
80 \\
85 \\
100\end{array}$ & $\begin{array}{r}55 \\
0 \\
0 \\
0\end{array}$ & $\begin{array}{r}0 \\
20 \\
15 \\
0\end{array}$ & $\begin{array}{r}80 \\
100 \\
100 \\
100\end{array}$ & $\begin{array}{r}20 \\
0 \\
0 \\
0\end{array}$ & $\begin{array}{l}100 \\
100 \\
100 \\
100\end{array}$ & $\begin{array}{l}0 \\
0 \\
0 \\
0\end{array}$ & $\begin{array}{l}\text { retardation } \\
\text { retardation } \\
\text { retardation } \\
\text { retardation }\end{array}$ \\
\hline \multicolumn{14}{|c|}{ Effects in Anthocidaris crassispina for comparison } \\
\hline $\begin{array}{l}\text { Running sea } \\
\text { water of } \\
\text { Laboratory }\end{array}$ & & $\begin{array}{l}95 \\
95 \\
\end{array}$ & $\begin{array}{l}5 \\
5\end{array}$ & $\begin{array}{l}95 \\
95 \\
\end{array}$ & $\begin{array}{l}0 \\
0 \\
\end{array}$ & $\begin{array}{l}0 \\
0\end{array}$ & $\begin{array}{l}100 \\
100 \\
\end{array}$ & $\begin{array}{l}0 \\
0 \\
\end{array}$ & $\begin{array}{l}0 \\
5 \\
\end{array}$ & $\begin{array}{r}100 \\
95 \\
\end{array}$ & & 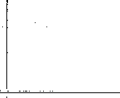 & $\begin{array}{l}\text { normal } \\
\text { normal }\end{array}$ \\
\hline $\begin{array}{l}\text { Water from } \\
\text { Tsunashirazu } \\
\text { cove }\end{array}$ & $\begin{array}{c}\text { Bottom } \\
(5 \mathrm{~m})\end{array}$ & $\begin{array}{l}90 \\
90\end{array}$ & $\begin{array}{l}10 \\
15\end{array}$ & $\begin{array}{l}90 \\
85\end{array}$ & $\begin{array}{l}0 \\
0\end{array}$ & $\begin{array}{l}15 \\
20\end{array}$ & $\begin{array}{l}85 \\
80\end{array}$ & $\begin{array}{l}0 \\
0\end{array}$ & $\begin{array}{l}10 \\
20\end{array}$ & $\begin{array}{l}90 \\
80\end{array}$ & $\cdots$ & & $\begin{array}{l}\text { almost normal } \\
\text { retardation }\end{array}$ \\
\hline
\end{tabular}




\section{Considerations}

It has already been reported by many researchers (Waterman 1937, Wilson and Armstrong 1951-1961, Bernhard 1957, Okubo and Okubo 1962, Soyer 1963, Bougis 1961-1967, Hagström and Lönning 1973 and Heslinga 1976) that the polluted sea water affects the pluteus formation in various sea urchins. Especially, Heslinga (1976) reports that the pluteus process appears to be very sensitive and therefore may be best suited to assess the stress by pollution in Echinometra.

Results of the present experiments with Anthocidaris and Peronella show clearly that the effects of various polluted sea water are more pronounced in these species, too, in pluteus formation than any other stages prior to it and that the whole developmental stages from fertilization to pluteus formation seem more sensitive in Peronella than in Anthocidaris, especially this is remarkable in the metamorphosis of Peronella.

All these were demonstrated by experiments not only with some known chemicals but also with the polluted sea water samples from around Hatakejima Island, as seen in five tables.

The metamorphosis of Peronella japonica Mortensen has been reported by Mortensen 1921, Onoda 1938, Okazaki and Dan 1954 and Okazaki 1975. In contrast with that it requires a long culture to bring the eggs of regular sea urchins to metamorphosis, in this species of sand dollars, the metamorphosis is completed in three to four days after fertilization. The larva of Peronella is shaped just like a pluteus, though with only two arms. It has no functional digestive tract and therefore does not need feeding till it completes metamorphosis. It is mentioned already that the number of arms may vary, from zero to four in the pluteus of Peronella; this seem however, to cause no essential differences in later stages, since all larvae with different number of arms complete the metamorphosis quite similarly (Okazaki and Dan 1954). It has been stated that this might be due partly to the influence of laboratory conditions (Mortensen 1921). In the present experiments, however, unusual numbers of pluteus arms less or more than two were mostly observed in company with retardation of development in the polluted sea water samples from around Hatakejima Island, and the plutei with such irregular numbers of arms were not able to complete the metamorphosis.

As seen in the present experiments, in marine pollution bioassay using fertilized sea urchin eggs, it is very necessary to check the later developmental stages as far as possible, at least the pluteus formation and even the metamorphosis if possible. However, it requires a long culture to do this in regular urchins, and actually it is requested that the bioassay is done in a short time. The significance of the results obtained by a short-time bioassay checking the earlier developmental stages from fertilization to gastrulation should be estimated properly on the background of more strict results that will be obtained by experiments with plutei.

Although the whole developmental stages to metamorphosis can be traced easily and in a short-time in Peronella and they are seemingly more sensitive to pollutants, 
according to Okazaki's view, Peronella is a very special urchin and therefore there may be some questions as to the generalization of the results obtained in this species. The author does not recommend the use of this sand dollar in the routine work of bioassay, but considers that the experiments with it may be useful as supplementary ones because of its rapid development and sharp sensitivity, especially in metamorphosis, to pollutants.

The author wishes to express his hearty thanks to the staff of the Seto Marine Biological Laboratory for the fund and facilities given to him in carrying out the researches at the Laboratory, particularly to Prof. T. Tokioka, the director of the Laboratory, for advices and criticism and for kindness in reading the manuscript. The author's deep gratitude is also due to Prof. Kayo Okazaki of Tokyo Metropolitan University for important suggestions and to Dr. Michio Imafuku and Mr. Tohoru Imaoka of the Laboratory for their help in obtaining the meterial used.

\section{Summary}

1. Effects of some pollutants upon the pluteus formation in the sea urchin, Anthocidaris crassispina, were examined.

2. Effects of some pollutants and polluted sea water upon the developmental stages from fertilization to metamorphosis in the sand dollar, Peronella japonica, were studied. 3. The effects were more pronounced in the pluteus formation than in any other stages prior to it in Anthocidaris and Peronella. Throughout the whole stages from fertilization to pluteus formation, Peronella was seemingly more sensitive than Anthocidaris, the sensitivity was especially remarkable in the metamorphosis of Peronella.

4. All these tendencies were demonstrated again in the tests with the polluted sea water samples from around Hatakejima Island.

5. Results in a short-time marine pollution bioassay checking the earlier developmental stages from fertilization to gastrulation should be estimated on the background of the more strict results that will be obtained by checking the later stages, at least the pluteus formation. The use of the metamorphosis of Peronella for bioassay may be valuable as a supplementary test, because of the rapid development and remarkable sensitivity in this sand dollar.

\section{REFERENCES}

Bernhard, M. 1957. Die Kultur von Seeigellarven (Arbacia lixula L.) in kunstlichen und naturlichen Meerwasser mit Hilfe von Ionenaustauschsubstanzén und Komplexbildnern. Publ. Sta. zool. Napoli 29: pp. 80-95.

Bougis, P. 1967. Utilisation des plutéus en écologie expérimentale. Helgoländer wiss. Meeresunters. 15: pp. 59-68.

Hagström, B.E. and Lönning, S. 1973. The sea urchin egg as a testing object in toxicology. Acta Pharmacol. et Toxicol. 32: Suppl. I. pp. 1-49.

Heslinga, G.A. 1976. Effects of copper on the coral-reef echinoid Echinometra mathaei. Mar. Biol. 35: pp. 155-160.

Kobayashi, N. 1971. Fertilized sea urchin eggs as an indicatory material for marine pollution bioas- 
say, preliminary experiments. Publ. Seto Mar. Biol. Lab. 18: pp. 379-406.

Kobayashi, N., Nogami, H. and Doi, K. 1972. Marine pollution bioassay by using sea urchin eggs in the Inland Sea of Japan (Seto-Naikai). Publ. Seto Mar. Biol. Lab. 19: pp. 359-381.

Kobayashi, N. 1974. Marine pollution bioassay by sea urchin eggs, an attempt to enhance accuracy. Publ. Seto Mar. Biol. Lab. 21 : pp. 377-391.

Mortensen, T. 1921. Studies of the development and larval forms of echinoderms. pp. 109-117. Copenhagen.

Okazaki, K. and Dan, K. 1954. The metamorphosis of partial larvae of Peronella japonica Mortensen, a sand dollar. Biol. Bull. 106: pp. 83-99.

Okazaki, K. 1975. Normal development to metamorphosis. In The sea urchin embryo. Ed. by Czihak, G.: pp. 177-232. Berlin.

Okubo, K. and Okubo, T. 1962. Study on the bio-assay method for the evaluation of water pollution-II. Use of the fertilized eggs of sea urchins and bivalves. Bull. Tokai Regional Fish. Res. Lab. 32: pp. 131-140.

Onoda, K. 1938. Notes on the development of some Japanese echinoids, with special reference to the structure of the larval body. Report III. Jap. J. Zool. 8: pp. 1-13.

Soyer, J. 1963. Contribution a l'étude des effets biologiques du mercure et de l'argent dans l'eau de mer. Vie et Milieu 14: pp. 1-36.

Waterman, A.J. 1937. Effects of salts of heavy metals on development of the sea urchin, Arbacia punctulata. Biol. Bull. 73: pp. 401-420.

Wilson, D.P. and Armstrong, F.A.J. 1961. Biological differences between sea waters: Experiments in 1960. J. Mar. Biol. Assoc. 41: pp. 663-681. 